\title{
Compactação do solo na cultura do feijoeiro. I: efeitos nas propriedades físico-hídricas do solo
}

\author{
Luís F. Stone ${ }^{1}$, Cleber M. Guimarães ${ }^{2}$ \& José A. A. Moreira ${ }^{3}$ \\ 1 Embrapa Arroz e Feijão, CP 179, CEP 75375-000, Santo Antônio de Goiás, GO. Fone: (62) 533-2186, Fax: (62) 533-2100. \\ E-mail: stone@cnpaf.embrapa.br (Foto) \\ 2 Embrapa Arroz e Feijão. E-mail: cleber@cnpaf.embrapa.br \\ 3 Embrapa Arroz e Feijão. E-mail: jaloisio@cnpaf.embrapa.br
}

Protocolo $170-5 / 12 / 2001$

\begin{abstract}
Resumo: O problema de compactação do solo vem aparecendo sistematicamente na região do Cerrado, onde os sistemas convencionais de manejo do solo têm causado desagregação excessiva da camada arável, o encrostamento superficial e a formação de camadas coesas ou compactadas (pé-de-grade ou pé-de-arado). Como alternativa, os agricultores adotaram o Sistema Plantio Direto; entretanto, em várias situações vêm sendo relatadas ocorrências de aumento da densidade do solo e diminuição da macroporosidade, o que tem sido diagnosticado como compactação, fazendo com que o agricultor seja obrigado a movimentar o solo. Este trabalho teve por objetivo verificar como a compactação do solo afeta as suas propriedades físicas e de transmissão de água. Para isto, utilizaram-se colunas de solo compactadas artificialmente, para produzir densidades do solo de 1,$0 ; 1,2 ; 1,4$ e $1,6 \mathrm{~kg} \mathrm{dm}^{-3}$. As propriedades de transmissão de água foram determinadas com infiltrômetros de tensão, para as cargas de pressão de $-0,5 ;-2,0$ e $-6,0 \mathrm{~cm}$ de água. 0 aumento da densidade do solo aumentou sua resistência à penetração e reduziu linearmente a porosidade total e a macroporosidade. Houve ainda, redução no tamanho dos poros para o fluxo de água, reduzindo a condutividade hidráulica do solo. Esta redução foi mais acentuada na maior carga de pressão testada. Com a carga de pressão de $-2,0 \mathrm{~cm}$ de água, a redução no tamanho dos poros foi parcialmente compensada com aumento do seu número. $O$ método do infiltrômetro de tensão mostrou-se adequado em distinguir diferenças nas propriedades de transmissão de água de um solo submetido a diferentes níveis de compactação.
\end{abstract}

Palavras-chave: Phaseolus vulgaris, infiltrômetro de tensão, condutividade hidráulica, porosidade do solo, densidade do solo

\section{Soil compaction in a bean crop. I: Effects on soil physical and water properties}

\begin{abstract}
Soil compaction is a problem in the Savanna region, where conventional soil tillage systems have caused excessive disaggregation of the arable layer, superficial crusts, and compacted subsuperficial layers. As an alternative, the farmers have adopted the no-tillage system. However, in various situations, an increase of soil bulk density and a decrease of macroporosity has been observed. This process is diagnosed as soil compaction and requires from the farmers the turnover of the soil. This work has the objective to verify how soil compaction affects physical and water transmission properties of soil. Artificially compacted soil columns were used to provide soil bulk densities of $1.0 ; 1.2 ; 1.4$; and $1.6 \mathrm{~kg} \mathrm{dm}^{-3}$. Water transmission properties were determined with tension infiltrometers at pressure heads of -0.5; -2.0 ; and $-6.0 \mathrm{~cm}$ of water column. Increasing soil bulk density increased soil resistance to penetration and reduced linearly soil porosity and macroporosity. The flow-weighted mean pore size was reduced too decreasing soil hydraulic conductivity. This reduction was more pronounced at higher pressure heads. The reduction in pore size was partially compensated with the increase in the number of pores, at a pressure head of $-2.0 \mathrm{~cm}$ of water. The method of tension infiltrometer was adequate to distinguish differences in water transmission properties of a soil submitted to different levels of compaction.
\end{abstract}

Key words: Phaseolus vulgaris, tension infiltrometer, hydraulic conductivity, soil porosity, bulk density 


\section{INTRODUÇÃO}

O termo compactação do solo refere-se à compressão do solo não saturado, durante a qual ocorre um aumento da densidade, em conseqüência da redução de volume pela expulsão do ar (Dias Jr. \& Pierce, 1996). Adensamento, por sua vez, é o fenômeno de deposição de partículas menores no espaço poroso, devido à desagregação da estrutura física do solo, causando aumento da massa de determinada porção do solo e mantendo constante o volume inicial, o que causa, também, aumento da densidade do solo, porém sem participação direta da pressão.

Pesquisadores têm demonstrado claramente o efeito da compactação nas propriedades físicas do solo. A compactação aumenta a densidade e sua resistência mecânica (Hillel, 1982; Moraes, 1984) mas diminui a porosidade total, o tamanho e a continuidade dos poros (Hillel, 1982; Moraes, 1984; Smucker \& Erickson, 1989). A compactação do solo pode ter efeitos benéficos, atribuídos à melhoria do contato solo-semente (Smucker \& Erickson, 1989) e ao aumento da disponibilidade de água em anos secos (Raghavan \& Mickyes, 1983). Entretanto, a compactação excessiva pode limitar a adsorção e/ou absorção de nutrientes, infiltração e redistribuição de água, trocas gasosas e desenvolvimento do sistema radicular (Smucker \& Erickson, 1989) resultando em decréscimo da produção, aumento da erosão e da potência necessária para o preparo do solo (Soane, 1990).

O problema de compactação/adensamento do solo vem aparecendo sistematicamente na região do Cerrado, onde os sistemas convencionais de manejo do solo promovem a desagregação excessiva da camada arável, o encrostamento superficial e a formação de camadas coesas ou compactadas, denominadas pé-de-grade ou pé-de-arado (Freitas, 1994). Como alternativa, os agricultores vêm adotando o Sistema Plantio Direto (SPD). Baseado na ausência de movimentação do solo e na manutenção de resíduos orgânicos na superfície do solo, o SPD altera a dinâmica da matéria orgânica e da atividade biológica modificando, principalmente, os processos intrínsecos do solo, provocando alterações na sua estrutura e na dinâmica físico-hídrica. Em várias situações, porém, têm sido relatadas ocorrências de aumento da densidade do solo e diminuição da macroporosidade com o uso do SPD. Este processo, diagnosticado como compactação devido ao fato de, supostamente, prejudicar o crescimento das raízes e o movimento vertical de água, faz com que o agricultor seja obrigado a movimentar o solo, desfazendo o trabalho biológico e físico de vários anos, destruindo a estrutura do solo, provoca a rápida mineralização da matéria orgânica e prejudica a atividade biológica.

Com a compactação, ocorrem reduções significativas, principalmente no volume de macroporos, enquanto os microporos permanecem praticamente inalterados (Hillel, 1982). Isto afeta a infiltração da água no solo, que está relacionada diretamente com a macroporosidade do solo. Os macroporos são também importantes para o crescimento das raízes (Wang et al., 1986) e para o movimento de solutos (Beven \& Germann, 1982). Assim, a medição e a caracterização da transmissão de água nos macroporos do solo podem ser usadas para avaliar o grau de compactação do solo. Para isto, a técnica do infiltrômetro de tensão é bastante promissora (Smettem \& Clothier, 1989; Reynolds \& Elrick, 1991). O método, in situ, requer um mínimo de perturbação do solo, é relativamente rápido e opera efetivamente na faixa de carga de pressão da água (P) dos poros, próximo de zero, ou faixa dos macroporos $(-15 \mathrm{~cm} \leq \mathrm{P} \leq 0 \mathrm{~cm}$ de água; Watson \& Luxmore, 1986) em que os macroporos do solo são considerados hidraulicamente ativos e importantes para a transmissão de água e solutos (Smettem \& Clothier, 1989; Ankeny et al., 1990; Clothier et al., 1992). Além disso, o método pode medir duas das mais significativas propriedades da transmissão de água dos macroporos do solo, chamadas condutividade hidráulica próximo da saturação e raio médio ponderado do poro, para fluxo (White et al., 1992). O uso desta técnica moderna possibilitaria o diagnóstico mais preciso do processo de adensamento e de compactação, permitindo que os problemas físicos do solo observados no plantio direto, sejam corrigidos dentro do sistema, com a rotação de culturas, o uso de culturas de cobertura (subsolagem biológica) e com uso de rompedores na semeadora, entre outros.

O objetivo deste trabalho foi verificar como a compactação do solo afeta as suas propriedades físicas e de transmissão de água.

\section{MATERIAL E MÉTODOS}

O estudo foi realizado em casa-de-vegetação da Embrapa Arroz e Feijão, em Santo Antônio de Goiás, GO, utilizando-se colunas de solo acondicionadas em tubos de PVC de $25 \mathrm{~cm}$ de diâmetro e $40 \mathrm{~cm}$ de altura. $\mathrm{O}$ solo utilizado foi um Latossolo Vermelho perférrico, cuja análise granulométrica, realizada pelo método da pipeta (Embrapa, 1997) apresentou teores de areia, silte e argila iguais a 430, $80 \mathrm{e} 490 \mathrm{~g} \mathrm{~kg}^{-1}$, respectivamente (classe textural argila). $\mathrm{O}$ solo foi secado ao ar, passado em peneiras com malha de $2 \mathrm{~mm}$ e, após, compactado dentro das colunas, nos primeiros $20 \mathrm{~cm}$, para produzir densidades do solo de 1,0; 1,$2 ; 1,4 \mathrm{e} 1,6 \mathrm{~kg} \mathrm{dm}^{-3}$. Utilizou-se uma prensa hidráulica para se estabelecer as densidades de 1,4 e 1,6 $\mathrm{kg} \mathrm{dm}^{-3}$. Em cada coluna foram colocadas seis sementes das cultivares de feijão Pérola ou BAT 477. Após a emergência das plântulas fez-se o desbaste, deixando-se três por coluna. Durante a execução do experimento, foram feitas irrigações para elevar o conteúdo de água do solo para a capacidade de campo $(-10 \mathrm{kPa})$ sempre que tensiômetros instalados a $10 \mathrm{~cm}$ de profundidade sinalizavam que o potencial da água do solo estava ao redor de $-35 \mathrm{kPa}$. Utilizou-se o delineamento experimental de blocos ao acaso, com parcelas subdivididas, com três repetições. As cultivares constituíram as parcelas, e as densidades do solo, as subparcelas. Neste trabalho, consideraram-se apenas as variáveis referentes às propriedades físicas e de transmissão de água do solo; as referentes às plantas, foram objeto de outro trabalho (Guimarães et al., 2002). Assim, para este trabalho o delineamento considerado foi o de blocos ao acaso, com seis repetições.

A determinação da microporosidade do solo foi realizada durante a floração do feijoeiro, em amostras indeformadas coletadas com cilindros de $5 \mathrm{~cm}$ de diâmetro por $5 \mathrm{~cm}$ de altura, enquanto amostras deformadas também foram retiradas para 
determinação da densidade de partículas. A microporosidade foi determinada pelo método da mesa de tensão, a densidade de partículas pelo método do balão volumétrico, a porosidade total pela relação entre a densidade do solo e a densidade de partículas, e a macroporosidade pela diferença entre a porosidade total e a microporosidade, utilizando-se a metodologia apresentada em Embrapa (1997). A resistência do solo à penetração $(\mathrm{R}), \mathrm{em} \mathrm{MPa}$, foi determinada por meio de um penetrômetro de bolso, convertendo a leitura (L) do mesmo, pela seguinte fórmula:

$$
\mathrm{R}=9,81 \mathrm{~L} /[0,7952(40-\mathrm{L})]^{2}
$$

A condutividade hidráulica do solo foi determinada com o auxílio de três infiltrômetros de disco, também chamados infiltrômetros de tensão (IT) com raio de $1,59 \mathrm{~cm}$, sendo um para cada carga de pressão considerada: $-0,5 ;-2,0$ e $-6,0 \mathrm{~cm}$ de água. Utilizou-se o método proposto por Zhang (1997) o qual requer a medição da infiltração acumulada versus tempo, cujos resultados foram ajustados pela função:

$$
\mathrm{I}=\mathrm{C}_{1} \mathrm{t}+\mathrm{C}_{2} \mathrm{t}^{0,5}
$$

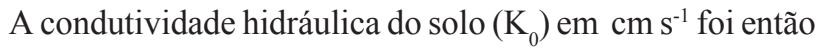
calculada por:

$$
\mathrm{K}_{0}=\mathrm{C}_{1} / \mathrm{A}_{0}
$$

$\mathrm{A}_{0}$ foi calculado utilizando-se a seguinte equação:

$$
A_{0}=\left[11,65\left(n^{0,1}-1\right) e^{[7,5(n-1,9)} \alpha^{\left.P_{0}\right]}\right] /(\alpha r)^{0,91}
$$

em que n e $\alpha$ são parâmetros de van Genuchten (1980) para o solo, $\mathrm{r}$ é o raio do disco e $\mathrm{P}_{0}$ é a carga de pressão na superfície do disco. Os parâmetros de van Genuchten foram obtidos do trabalho de Carsel \& Parrish (1988) considerando-se a textura argilosa do solo utilizado. Os valores de $\mathrm{A}_{0}$ calculados foram, respectivamente, iguais a 5,2; 4,9 e 4,0 para as diferentes cargas de pressão consideradas.

$O$ raio médio ponderado para fluxo, $\mathrm{R}_{0}(\mathrm{~cm})$ como definido por Philip (1987) foi calculado por:

$$
\mathrm{R}_{0}=\left[\sigma \mathrm{K}_{0}\right] /\left[\rho g \mathrm{M}_{0}\right]
$$

em que $\sigma\left(\mathrm{g} \mathrm{s}^{-2}\right)$ é a tensão superficial da água, $\rho\left(\mathrm{g} \mathrm{cm}^{-3}\right)$ é a densidade da água, $g\left(\mathrm{~cm} \mathrm{~s}^{-2}\right)$ é a aceleração da gravidade e $\mathrm{M}_{0}$ $\left(\mathrm{cm}^{2} \mathrm{~s}^{-1}\right)$ é o potencial de fluxo mátrico do solo.

$\mathrm{O}$ valor de $\mathrm{M}_{0}$ é definido por (Gardner, 1958):

$$
\left.\mathrm{M}_{0}=\int \underset{\mathrm{P}_{\mathrm{i}}}{\mathrm{K}} \stackrel{\mathrm{P}_{0}}{\mathrm{P}}\right) d \mathrm{P} \mathrm{P}_{\mathrm{i}} \leq \mathrm{P} \leq \mathrm{P}_{0} \leq 0
$$

sendo $\mathrm{K}(\mathrm{P})\left(\mathrm{cm} \mathrm{s}^{-1}\right)$ a relação condutividade hidráulica $(\mathrm{K})$ carga de pressão $(\mathrm{P})$ para o umedecimento do solo, e $\mathrm{P}_{\mathrm{i}}(\mathrm{cm})$ a carga inicial de pressão da água do poro no solo insaturado, no momento da medição com o IT.
As estimativas de $\mathrm{M}_{0}$ foram obtidas pela divisão da integral de $\mathrm{M}_{0}$ nos componentes 'tensão' e potencial de fluxo 'residual', $\mathrm{M}_{\mathrm{n}}$ e $\mathrm{M}_{\mathrm{r}}$, respectivamente.

$$
\mathrm{M}_{0}=\mathrm{M}_{\mathrm{n}}+\mathrm{M}_{\mathrm{r}}
$$

donde

$$
\mathrm{M}_{0}=\int \underset{\mathrm{P}_{0}}{\mathrm{~K}} \stackrel{\left.\stackrel{\mathrm{P}_{\mathrm{n}}}{\mathrm{P}}\right)}{\mathrm{P}_{\mathrm{n}}} \mathrm{PP}_{\mathrm{n}} \leq \mathrm{P} \leq \mathrm{P}_{0}
$$

e

$$
\mathrm{M}_{0}=\int \underset{\mathrm{P}_{\mathrm{i}}}{\mathrm{K}} \stackrel{\left.\stackrel{\mathrm{P}_{\mathrm{n}}}{P}\right)}{\mathrm{P}} \mathrm{P}_{\mathrm{i}} \leq \mathrm{P} \leq \mathrm{P}_{\mathrm{n}}
$$

Os valores de $\mathrm{M}_{\mathrm{n}}$ foram determinados pela integração numérica sob os segmentos de reta ajustados aos dados de $\mathrm{K}_{0}$ versus $\mathrm{P}_{0}$. Os valores de $\mathrm{M}_{\mathrm{r}}$ foram determinados pelo rearranjo da equação que descreve o fluxo constante de um infiltrômetro de tensão (Wooding, 1968):

$$
\mathrm{Q}_{0}=\left[\pi \mathrm{r}^{2} \mathrm{~K}_{0}\right]+\left[\mathrm{GrM}_{0}\right]
$$

em que $\mathrm{Q}_{0}\left(\mathrm{~cm}^{3} \mathrm{~s}^{-1}\right)$ é a taxa de fluxo constante para fora do IT e para dentro do solo insaturado, quando uma carga de pressão constante, $\mathrm{P}_{0}$ (cm de água), é aplicada à membrana do IT, $\mathrm{r}(\mathrm{cm})$ é o raio da superfície de infiltração (solo) que recebe água de IT, no caso o raio do disco do infiltrômetro, $\mathrm{K}_{0}\left(\mathrm{~cm} \mathrm{~s}^{-1}\right)$ é a condutividade hidráulica próximo da saturação do solo à $\mathrm{P}_{0}, \mathrm{G}$ é um fator de forma adimensional constante com um valor de 4,219 (Reynolds \& Elrick, 1991) e $\mathrm{M}_{0}\left(\mathrm{~cm}^{2} \mathrm{~s}^{-1}\right)$ é o potencial de fluxo mátrico do solo. Assim:

$$
\mathrm{M}_{\mathrm{r}}=\left[\mathrm{Q}_{\mathrm{n}}-\pi \mathrm{r}^{2} \mathrm{~K}_{\mathrm{n}}\right] /[\mathrm{Gr}]
$$

sendo: $\mathrm{Q}_{\mathrm{n}}=\mathrm{Q}_{0}$ e $\mathrm{K}_{\mathrm{n}}=\mathrm{K}_{0}$ a $\mathrm{P}_{0}=\mathrm{P}_{\mathrm{n}}$.

A concentração de poros $\mathrm{R}_{0}, \mathrm{~N}_{0}$ (número de poros $\mathrm{m}^{-2}$ ), foi estimada usando-se a lei de Poiseuille para fluxo em um tubo capilar.

$$
\mathrm{N}_{0}=\left[8 \mu \mathrm{K}_{0}\right] /\left[\rho g \pi \mathrm{R}_{0}{ }^{4}\right]
$$

donde $\mu\left(\mathrm{g} \mathrm{cm}^{-1} \mathrm{~s}^{-1}\right)$ é a viscosidade dinâmica da água.

Os dados obtidos foram submetidos às análises de variância e de regressão.

\section{RESULTADOS E DISCUSSÃO}

Os valores da porosidade total $(\mathrm{P})$, da macro $(\mathrm{Mp})$ e da microporosidade $(\mathrm{mp})$ e da resistência do solo à penetração (R) obtidos em resposta às diferentes densidades do solo (ds) 
estabelecidas, encontram-se na Tabela 1. Esses valores são semelhantes aos obtidos por Alvarenga et al. (1996) para os mesmos níveis de compactação do solo. A porosidade e a macroporosidade decresceram linearmente com o aumento da densidade do solo, segundo as equações $\mathrm{P}=1-0,374 \mathrm{ds}$ $\left(\mathrm{R}^{2}=1^{* *}\right)$ e $\mathrm{Mp}=0,72-0,424 \mathrm{ds}\left(\mathrm{R}^{2}=0,99^{*}\right)$, respectivamente. Alvarenga et al. (1996) e Merotto Jr. \& Mundstock (1999) também verificaram decréscimo em $\mathrm{P}$ e $\mathrm{Mp}$ com o aumento da densidade do solo. A microporosidade não foi afetada significativamente pela densidade do solo, apesar da tendência de aumento com o seu incremento, concordando com os resultados obtidos por Merotto Jr. \& Mundstock (1999). A pressão mecânica exercida sobre os agregados pela compactação deve ter provocado a sua ruptura, facilitando a aproximação das partículas, cuja conseqüência imediata foi a redução da porosidade total, pela diminuição dos poros maiores e com algum aumento no número de poros menores. Trabalhos têm mostrado que, quando a macroporosidade do solo é reduzida a valores inferiores a $0,15 \mathrm{~m}^{3} \mathrm{~m}^{-3}$, o crescimento das raízes é prejudicado (Meredith \& Patrick Jr., 1961; Cintra et al., 1983). Neste caso, só a partir de ds $=1,4 \mathrm{~kg} \mathrm{dm}^{-3}$ ocorreria problema para as raízes. Além disto, ao diminuir a macroporosidade, parte significativa da água fica retida nos microporos, sob altas tensões, indisponível para as plantas (Kertzmann, 1996).

Tabela 1. Porosidade e resistência do solo à penetração em relação à densidade do solo

\begin{tabular}{ccccc}
\hline \multirow{2}{*}{$\mathrm{ds}^{*}\left(\mathrm{~kg} \mathrm{dm}^{-3}\right)$} & \multicolumn{3}{c}{ Porosidade $\left(\mathrm{m}^{3} \mathrm{~m}^{-3}\right)$} & \multirow{2}{*}{$\mathrm{R}^{* *}(\mathrm{MPa})$} \\
\cline { 2 - 4 } & Total & Macro & Micro & \\
\hline 1,0 & 0,625 & 0,288 & 0,337 & 0,1 \\
1,2 & 0,550 & 0,220 & 0,330 & 1,0 \\
1,4 & 0,476 & 0,131 & 0,345 & 1,7 \\
1,6 & 0,401 & 0,036 & 0,365 & 6,1 \\
\hline
\end{tabular}

* ds - densidade do solo

** $\mathrm{R}$ - resistência do solo à penetração

A resistência do solo à penetração aumentou de maneira quadrática com a densidade do solo $\left(\mathrm{R}=25,9-47,5 \mathrm{ds}+21,9 \mathrm{ds}^{2}\right.$, $\mathrm{R}^{2}=0,96^{*}$ ) devido ao fato de que, na densidade de $1,6 \mathrm{~kg} \mathrm{dm}^{-3}$, a macroporosidade do solo era muito pequena. Merotto Jr. \& Mundstock (1999) e Rosolem et al. (1999) verificaram aumento exponencial da resistência do solo à penetração, com o incremento da densidade do solo. Tormena \& Roloff (1996) afirmam que valores de resistência do solo à penetração iguais ou maiores que $2 \mathrm{MPa}$ ão restritivos ao crescimento das raízes e que o valor de $1 \mathrm{MPa}$ é crítico, mas não impeditivo. Merotto Jr. \& Mundstock (1999) também não encontraram restrições ao crescimento das raízes de trigo até o valor de $2 \mathrm{MPa}$. Entretanto, Rosolem et al. (1999) verificaram que a resistência do solo à penetração igual a 1,3 MPa reduziu pela metade o crescimento das raízes seminais adventícias do milho, corroborando com o discutido em relação à macroporosidade, de que, a partir de $\mathrm{ds}=1,4 \mathrm{~kg} \mathrm{dm}^{-3}$, haveria problema para o crescimento radicular.

A condutividade hidráulica decresceu com o aumento da densidade do solo (Fig. 1) sendo a redução maior na carga de pressão de $-0,5 \mathrm{~cm}$ de água, reforçando o conceito de que os maiores poros, em oposição aos menores, são mais facilmente destruídos com a compactação (Ankeny et al., 1990). Silva et

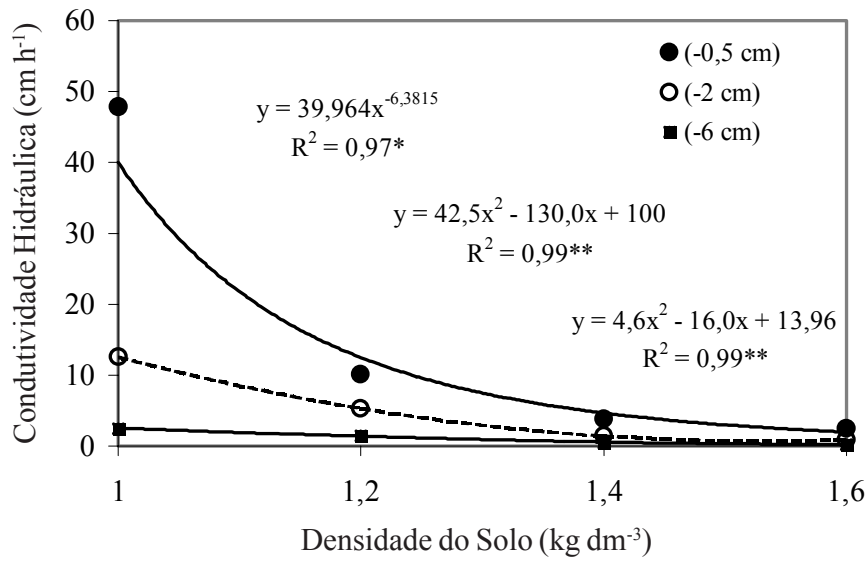

Figura 1. Condutividade hidráulica do solo, determinada sob diferentes cargas de pressão, em função da densidade do solo

al. (1986) observaram queda acentuada no valor da condutividade hidráulica saturada com a elevação do nível de compactação em dois Latossolos. Verifica-se, ainda, que os valores da condutividade hidráulica nas densidades de 1,4 e $1,6 \mathrm{~kg} \mathrm{dm}^{-3}$ são muito parecidos, sugerindo que o tamanho de poros para fluxo de água deva ser similar, ou seja, embora a compactação reduza o número de poros condutores de água em todas as cargas de pressão consideradas, ela destrói menor porcentagem de poros menores e maior porcentagem de poros maiores; isto pode ser comprovado na Figura 2, em que se observa que o raio médio ponderado para fluxo $\left(\mathrm{R}_{0}\right)$ diminuiu com o aumento da densidade do solo e que a redução foi mais acentuada (ajuste quadrático) para a carga de pressão de $-0,5 \mathrm{~cm}$ de água. Observa-se, ainda na Figura 1 , que os valores da condutividade hidráulica decresceram a medida em que as cargas de pressão estabelecidas nas membranas dos infiltrômetros, diminuíram. A redução na carga de pressão aplicada ao solo não compactado $\left(\mathrm{ds}=1 \mathrm{~kg} \mathrm{dm}^{-3}\right)$ causou maior decréscimo (cerca de 19 vezes) na condutividade hidráulica que nos compactados (entre 6,7 e 11,8 vezes) sugerindo que os macroporos maiores estão transportando maior proporção do fluxo total de água do solo não compactado, em relação ao compactado.

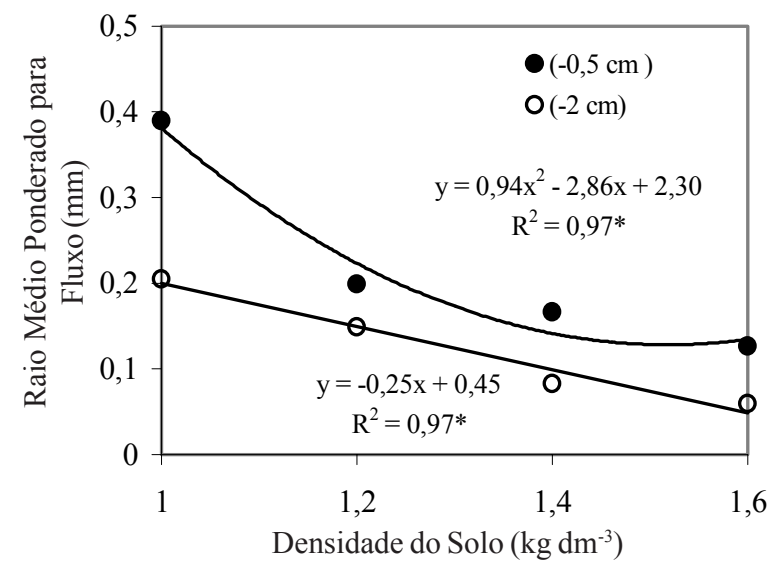

Figura 2. Raio médio ponderado para fluxo determinado sob duas cargas de pressão, em função da densidade do solo 


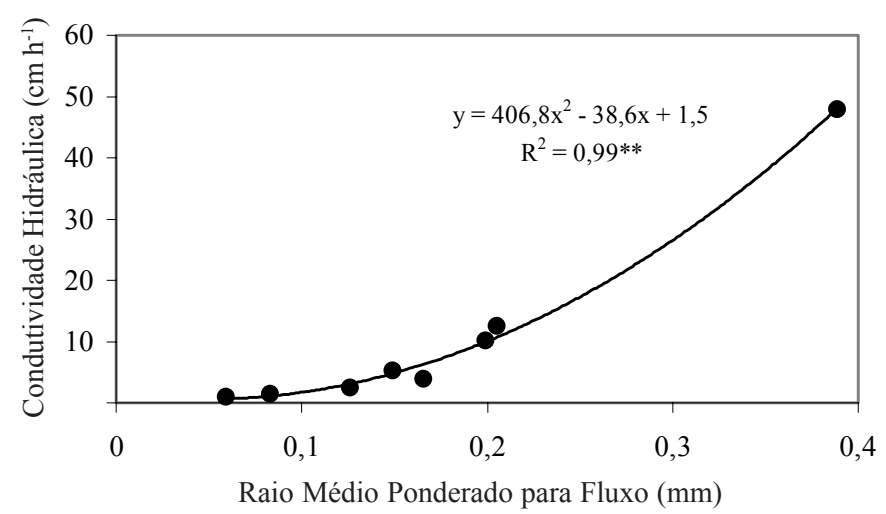

Figura 3. Condutividade hidráulica em função do raio médio ponderado para fluxo

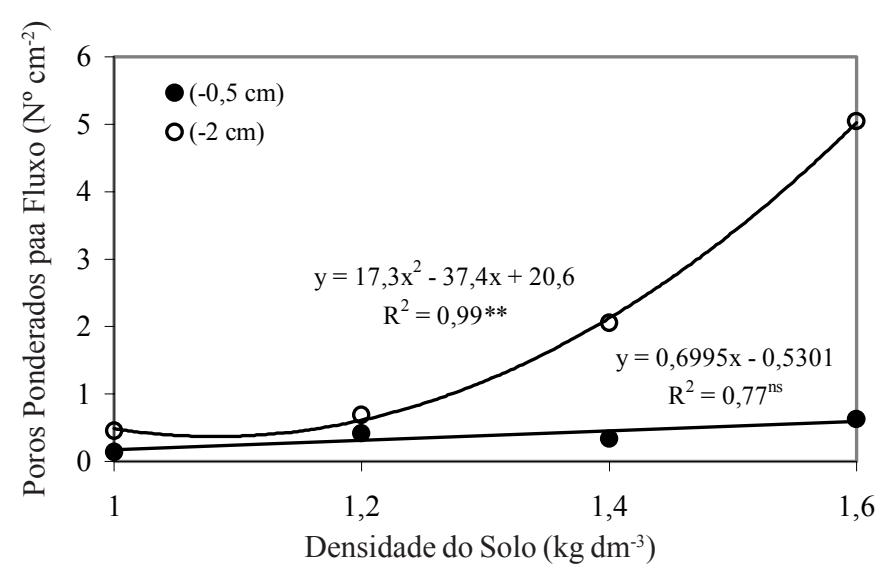

Figura 4. Número de poros ponderados para fluxo determinado sob duas cargas de pressão, em função da densidade do solo

O efeito da compactação na redução do tamanho dos poros, que efetivamente transportam água, explica a redução da condutividade hidráulica com o aumento da densidade do solo, pois ela é altamente relacionada com $\mathrm{R}_{0}$ (Fig. 3) reduzindo, de forma acentuada, o seu valor, com a redução do tamanho dos poros.

Pode-se observar na Figura 4, que a redução em $\mathrm{R}_{0}$ foi parcialmente compensada pelo aumento no número de poros por área $\left(\mathrm{N}_{0}\right)$ para a carga de pressão de $-2,0 \mathrm{~cm}$. Assim, embora o tamanho dos poros tenha diminuído com o aumento na densidade do solo, o número de poros por área aumentou. $\mathrm{Na}$ maior carga de pressão isto não ocorreu, não sendo significativa a relação entre $\mathrm{N}_{0}$ e a densidade do solo; a redução no tamanho dos poros, entretanto, pode restringir o desenvolvimento radicular. Sob a maior carga de pressão, o raio médio para fluxo no tratamento sem compactação ficou ao redor de $0,40 \mathrm{~mm}$, enquanto no mais compactado ficou ao redor de $0,15 \mathrm{~mm}$. Vepraskas \& Miner (1986) verificaram que a maior proporção de raízes que crescem dentro de macroporos, ocorreu em solos que apresentaram maiores quantidades e continuidade de macroporos. Assim, é importante o solo possuir poros grandes, o suficiente para permitir a entrada inicial das raízes das plantas.

\section{CONCLUSÕES}

1. A porosidade total e a macroporosidade diminuíram linearmente e a resistência do solo à penetração aumentou de maneira quadrática com o aumento da densidade do solo.
2. O aumento da densidade do solo reduziu o tamanho dos poros para fluxo de água e a condutividade hidráulica do solo. Esta redução foi mais acentuada com a maior carga de pressão testada.

3. Com a carga de pressão de $-2,0$ cm de água, a redução no tamanho dos poros foi parcialmente compensada com o aumento no número de poros.

4. O método do infiltrômetro de tensão mostrou-se adequado em distinguir diferenças nas propriedades de transmissão de água do solo submetido a diferentes níveis de compactação.

\section{LITERATURA CITADA}

Alvarenga, R.C.; Costa, L.M.; Moura Filho, W.; Regazzi, A.J. Crescimento de raízes de leguminosas em camadas de solo compactadas artificialmente. Revista Brasileira de Ciência do Solo, Campinas, v.20, p.319-326, 1996.

Ankeny, M.D.; Kaspar, T.C.; Horton, R. Characterization of tillage and traffic effects on unconfined infiltration measurements. Soil Science Society of America Journal, Madison, v.54, p.837-840, 1990.

Beven, K.J.; Germann, R.F. Macropores and water flow in soils. Water Resources Research, Washington, v.18, p.1311-1325, 1982.

Carsel, R.F.; Parrish, R.S. Developing joint probability distributions of soil water retention characteristics. Water Resources Research, Washington, v.24, p.755-769, 1988.

Cintra, F.L.D.; Mielniczuk, J.; Scopel, I. Caracterização do impedimento mecânico em um Latossolo Roxo do Rio Grande do Sul. Revista Brasileira de Ciência do Solo, Campinas, v.7, p.323-327, 1983.

Clothier, B.E.; Kirkham, M.B.; Mclean, J.E. In situ measurements of the effective transport volume for solute moving through soil. Soil Science Society of America Journal, Madison, v.56, p.733-736, 1992.

Dias Junior, M. de S.; Pierce, F.J. O processo de compactação do solo e sua modelagem. Revista Brasileira de Ciência do Solo, Campinas, v.20, p.175-182, 1996.

EMBRAPA - Empresa Brasileira de Pesquisa Agropecuária. Centro Nacional de Pesquisa de Solos (Rio de Janeiro, RJ). Manual de métodos de análise de solos. 2.ed. Rio de Janeiro: EMBRAPA-CNPS, 1997. 212p. EMBRAPA-CNPS. Documentos, 1

Freitas, P.L. de. Aspectos físicos e biológicos do solo. In: Landers, J.N. (ed.). Fascículos sobre experiências em plantio direto nos Cerrados. Uberlândia: APDC, 1994. Cap.9. p.187-196.

Gardner, W.R. Some steady-state solutions of the unsaturated moisture flow equation with application to evaporation from a water table. Soil Science, Baltimore, v.85, p.228-232, 1958.

Guimarães, C.M.; Stone, L.F.; Moreira, J.A.A. Compactação do solo na cultura do feijoeiro. II. Efeito sobre o desenvolvimento radicular e da parte aérea. Revista Brasileira de Engenharia Agrícola e Ambiental, Campina Grande, v.6, n.2, p.213-218, 2002.

Hillel, D. Introduction to soil physics. San Diego: Academic Press, 1982. 264p. 
Kertzmann, F.F. Modificações na estrutura e no comportamento de um Latossolo Roxo, provocadas pela compactação. São Paulo: USP, 1996. 153p. Tese Doutorado.

Meredith, H.L.; Patrick Jr., W.H. Effects of soil compaction on subsoil root penetration and physical properties of three soils in Louisiana. Agronomy Journal, Madison, v.53, p.163$167,1961$.

Merotto Jr., A.; Mundstock, C.M. Wheat root growth as affected by soil strength. Revista Brasileira de Ciência do Solo, Viçosa, v.23, n.2, p.197-202, 1999.

Moraes, W.V. de. Comportamento de características e propriedades físicas de um Latossolo Vermelho-Escuro, submetido a diferentes sistemas de cultivo. Lavras: UFLA, 1984. 107p. Dissertação Mestrado

Philip, J.R. The quasilinear analysis, scattering analog, and other aspects of infiltration and seepage. In: Fok, Y.S. (ed.). Infiltration development and application. Honolulu: Water Resources Research Center, 1987. p.1-27.

Raghavan, G.S.V.; Mickyes, E. Physical and hydraulic characteristics in compacted clay soils. Journal of Terramechanics, Elmsford, v.19, p.235-242, 1983.

Reynolds, W.D.; Elrick, D.E. Determination of hydraulic conductivity using a tension infiltrometer. Soil Science Society of America Journal, Madison, v.55, p.633-639, 1991.

Rosolem, C.A.; Fernandez, E.M.; Andreotti, M.; Crusciol, C.A.C. Crescimento radicular de plântulas de milho afetado pela resistência do solo à penetração. Pesquisa Agropecuária Brasileira, Brasília, v.34, n.5, p.821-828, 1999.

Silva, A.P. de; Libardi, P.L.; Camargo, O.A. Influência da compactação nas propriedades físicas de dois Latossolos. Revista Brasileira de Ciência do Solo, Campinas, v.10, n.2, p.91-95, 1986.

Smettem, K.R.J.; Clothier, B.E. Measuring unsaturated sorptivity and hydraulic conductivity using multiple disc permeameters. Journal of Soil Science, Oxford, v.40, p.563-568, 1989.
Smucker, A.J.M.; Erickson, A.E. Tillage and compactive modifications of gaseous flow and soil aeration. In: Larson, W.E.; Blake, G.R.; Allmaras, R.R.; Voorhees, W.B.; Gupta, S.C. (eds.). Mechanics related process in structured agricultural soils. NATO applied sciences. Amesterdam: Kluwer Academic Publishers, 1989. v.172, p.205-221.

Soane, B.D. The role of organic matter in soil compatibility: A review of some practical aspects. Soil \& Tillage Research, Amsterdam, v.16, p.179-201, 1990.

Tormena, C.A.; Roloff, G. Dinâmica da resistência à penetração de um solo sob plantio direto. Revista Brasileira de Ciência do Solo, Campinas, v.20, n.2, p.333-339, 1996.

van Genuchten, M.T.H. A closed-form equation for predicting the hydraulic conductivity of unsaturated soils. Soil Science Society of America Journal, Madison, v.44, p.892-898, 1980.

Vepraskas, M.J.; Miner, G.S. Effects of subsoiling and mechanical impedance on tobacco root growth. Soil Science Society of America Journal, Madison, v.50, p.423-427, 1986.

Wang, J.; Hesketh, J.D.; Woolley, J.T. Preexisting channels and soybean rooting patterns. Soil Science, Baltimore, v.141, p.432-437, 1986.

Watson, K.W.; Luxmore, R.J. Estimating macroporosity in a forest watershed by use of a tension infiltrometer. Soil Science Society of America Journal, Madison, v.50, p.578-582, 1986.

White, I.; Sully, M.J.; Perroux, K.M. Measurement of surfacesoil hydraulic properties: disk permeameters, tension infiltrometers, and other techniques. In: Topp, G.C.; Reynolds, W.D.; Green, R.E. (eds.). Advances of soil physical properties: Bringing theory into practice. Madison: American Society of Agronomy, 1992. p.69-103. Special Publication 30

Wooding, R.A. Steady infiltration from a shallow circular pond. Water Resources Research, Washington, v.4, p.1259-1273, 1968.

Zhang, R. Determination of soil sorptivity and hydraulic conductivity from the disk infiltrometer. Soil Science Society of America Journal, Madison, v.61, p.1024-1030, 1997. 